\title{
Optical propagation through a homogeneous turbulent shear flow
}

\author{
By C. RANDALL TRUMAN† AND MOON J. LEEł
}

Effects of organized turbulent structures on the propagation of an optical beam in a homogeneous shear flow have been studied. A passive-scalar field in a computed turbulent shear flow is used to represent index-of-refraction fluctuations, and phase errors induced in a coherent optical beam by turbulent fluctuations are computed. The organized vortical structures produce a scalar distribution with elongated regions of intense fluctuations which have an inclination with respect to the mean flow similar to that of the characteristic hairpin eddies. It is found that r.m.s. phase error is minimized by propagating approximately normal to the inclined vortical structures. Two-point correlations of vorticity and scalar fluctuation suggest that the regions of intense scalar fluctuation are produced primarily by the hairpin eddies.

\section{Introduction}

The passage of coherent electromagnetic beams through turbulent flow fields results in degradation of optical quality. Distortions in phase by index-of-refraction fluctuations reduce beam intensity in the far field. Propagation of wave in atmospheric situations has been studied extensively (see Tatarskii 1971, Chapter 4). The present study, however, is motivated by the need to understand (and predict) losses in optical quality induced by thin shear layers through which a beam must pass. These include, for example, a mixing layer at the exit cavity of a laser (see Baxter, Truman \& Masson 1988).

The physical problem of interest in the present study is a coherent optical beam in the visible range, whose width is large compared to the length scales of turbulence in the shear layer through which it passes. The beam width is assumed to be the same order as the size of the computational flow field, which is about ten times the largest length scale of turbulence. The beam wavelength selected was $4 \times 10^{-7} \mathrm{~m}$ with flow field dimensions on the order of $0.1 \mathrm{~m}$.

The effect of turbulent fluctuations upon optical quality is commonly modeled assuming isotropic, homogeneous turbulence with a Gaussian distribution (e.g. Tatarskii 1971, $§ 47-49)$. As noted by Liepmann (1979), however, "the theory of homogeneous [and isotropic] turbulence does not lead to decisive progress in coping with the shear flow and general mixing problems." Current understanding of turbulent shear flow shows the statistical theories, including models of optical

$\dagger$ University of New Mexico

$\ddagger$ Center for Turbulence Research 
degradation (see Sutton 1969), to be inadequate. Thus successful predictions of beam degradation must account for instantaneous turbulence structures of both the large- and small-scale eddies.

This work has been undertaken to gain an understanding of the relative importance of the large- and small-scale turbulent fluctuations in optical propagation. A passive-scalar field in a homogeneous shear flow computed by Rogers, Moin \& Reynolds (1986) is used to represent an instantaneous index-of-refraction field. It is our primary interest to explore the effects of organized turbulence structures in turbulent shear flow on the propagation of a coherent optical beam. The basis for computing phase error induced by turbulent fluctuations is discussed first.

\section{Wave propagation in a turbulent medium}

\subsection{Parabolic wave equation}

Maxwell's equations govern the behavior of an electromagnetic beam propagating through a turbulent medium. The magnetic permeability is assumed to be constant while the dielectric constant (and thus the refractive index) is assumed to be space-variant (Goodman 1985, pp. 393-399), and the effects of depolarization are negligible (Monin \& Yaglom 1975, Chapter 9). We neglect the time-dependence of the refractive index, since the time scale for propagation of light through a flow field is much smaller than that of turbulent fluctuations.

A scalar electric field $E\left(x_{*}\right)$ may be considered and the transformation $E=$ $u e^{i K x *}$ yields

$$
2 i K \frac{\partial u}{\partial x_{*}}+\nabla_{*}^{2} u+K^{2}\left(n^{2}-1\right) u=0
$$

where $x_{*}$ is the distance in the direction of propagation, $\nabla_{*}^{2}=\partial^{2} / \partial x_{*}^{2}+\partial^{2} / \partial y_{*}^{2}+$ $\partial^{2} / \partial z_{*}^{2}, K$ is the wavenumber of the coherent optical beam and $n\left(\mathbf{x}_{*}\right)$ is the index of refraction. The complex function $u\left(x_{*}\right)$, which represents the amplitude and the phase of the electric field, is then slowly varying in the propagation direction.

In practice, the time-averaged spatial variation in index of refraction is ignored. Such variations may displace the beam focus (e.g. beam steering), but induce no loss in far-field intensity (Born \& Wolf 1975, pp. 462-463). Moreover, the effect of these variations can be treated by conventional optical techniques.

Since the effects of turbulent structures are of primary interest, a fluctuating wave equation is described. The instantaneous index of refraction is written as

$$
n=1+n^{\prime}
$$

where $n^{\prime}$, typically of order $O\left(10^{-6}\right)$, is understood to be the fluctuation with respect to the spatial distribution of index of refraction.

If backscatter is not important, one may neglect the second derivative with respect to $x_{*}$ in (1). Then, after neglecting the term quadratic in $n^{\prime}$, one obtains the parabolized Helmholtz equation:

$$
2 i K \frac{\partial u}{\partial x_{*}}+\frac{\partial^{2} u}{\partial y_{*}^{2}}+\frac{\partial^{2} u}{\partial z_{*}^{2}}+2 K^{2} n^{\prime} u=0
$$


A pseudospectral method was developed by Clark, Truman \& Masson (1988) to solve (3) for the phase and amplitude of a coherent optical beam which propagates through a turbulent medium with a deterministic index-of-refraction field.

\subsection{Optical path difference}

When diffraction of the optical beam is significant, the parabolized Helmholtz equation (3) must be solved. For the present study, however, the selected parameters ensure that diffraction is negligible, and the restrictions of geometric optics (or acoustics) hold (Keller 1954). With the second derivatives representing diffraction neglected, the parabolic equation (3) reduces to an ordinary differential equation with a variable coefficient $n^{\prime}\left(x_{*}\right)$. Since changes in beam amplitude are negligible over a short propagation distance, only changes in phase need to be considered.

In this case, the phase error can be determined by

$$
\Delta \phi=K \int_{0}^{\bullet} n^{\prime} d x_{*}
$$

where $s$ is the distance along the propagation path. This integral, known as the optical path difference, multiplied by the wavenumber of the beam yields the phase error induced by variations in the index of refraction. The use of the optical path difference to compute phase errors along arbitrary paths allows the use of the full numerical database, which is beneficial in analyzing statistical quantities and associated instantaneous turbulence structures.

\section{Results and discussion}

A flow field in a homogeneous turbulent shear flow computed on a $128 \times 128 \times 128$ grid by Rogers, Moin \& Reynolds (1986) has been analyzed to provide 'data' for the present study. We have chosen a flow field (C128U12 in Rogers et al.'s notation) at dimensionless time $S t=12$ ( $S$ is the shear rate), in which most turbulence statistical correlations are 'fully-developed.' Here, $(x, y, z)$ denote the coordinates in the streamwise, transverse and spanwise directions, respectively.

\subsection{Passive-scalar field}

The instantaneous passive scalar $\theta\left(\theta_{2}\right.$ in Rogers et al.'s notation) subjected to uniform mean velocity and scalar gradients transverse to the mean flow, $d U / d y$ and $d \Theta / d y$, is used to represent the index-of-refraction fluctuations $n^{\prime}$; the passive scalar is scaled to an r.m.s. value of $10^{-6}$. As discussed in $\$ 2.1$, the mean scalar gradient is neglected, since only phase distortions due to turbulent fluctuations are of interest. The phase errors are computed based on the instantaneous index of refraction as determined from the passive scalar distribution.

In order to examine the spatial distribution, contours of the scalar fluctuations are shown on a vertical $x y$-plane in figure 1 . It is clearly shown that the scalar field consists of regions of intense fluctuation which have an orientation similar to the structures of the vorticity field, i.e. the hairpin vortices (Rogers \& Moin 1987). Notice that regions in which scalar fluctuations are two or even three times as large 


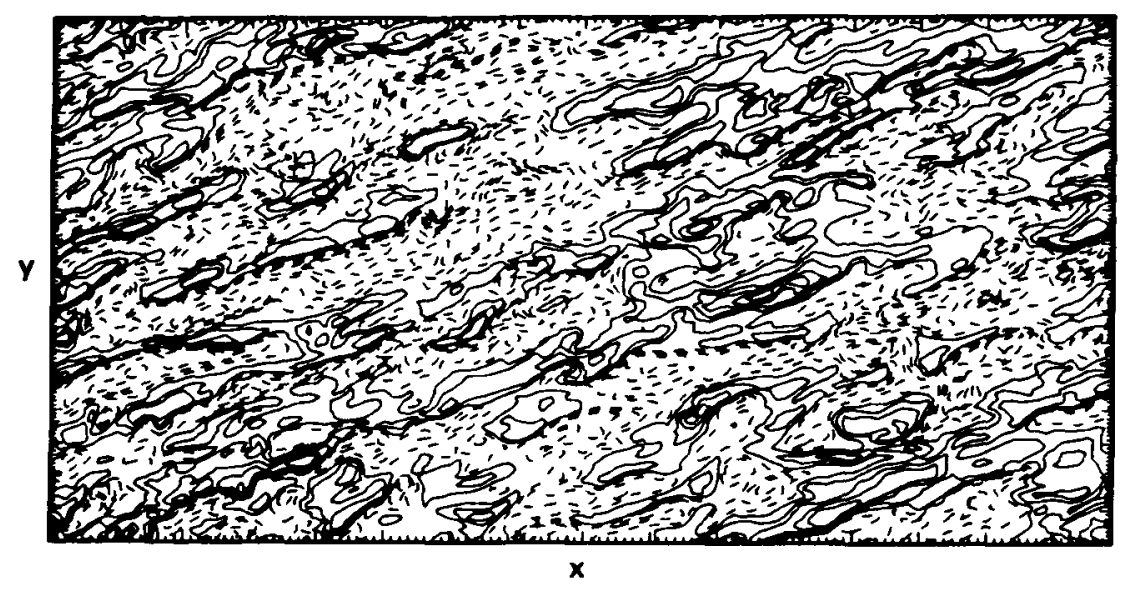

FIgURE 1. Contours of constant scalar fluctuation $\theta$ on an $x y$-plane in homogeneous turbulent shear flow (Rogers et al. 1986). The scalar field is elongated in the flow direction due to applied shear, similar to the vorticity field.

as the r.m.s. value are inclined at approximately $45^{\circ}$ from the gradient direction and are elongated along the direction of inclination. This result suggests that the large-scale vortical structures strongly influence the scalar distribution.

\subsection{Effect of propagation angle on phase error}

The nonisotropic nature of the scalar distribution immediately leads to a hypothesis that the phase errors depend on the direction of propagation through the turbulent field. In order to verify this hypothesis, phase errors have been computed by using (4) for propagation along several directions in the computed flow field. [For the present problem, solutions from (3) are almost identical to those from (4).] The angle of the beam propagation $\alpha$ is measured counter-clockwise from the flow direction ( $x$-axis); for example, propagation in the direction of the mean scalar gradient (y-axis) has an angle $\alpha=90^{\circ}$. Note that propagation at $\alpha-180^{\circ}$ and $\alpha$ are identical.

Figures $2(a-d)$ show contours of the phase error for propagation between the top and bottom $x z$-planes of the computational domain at $\alpha=45^{\circ}, 90^{\circ}, 135^{\circ}$ and $153.4^{\circ}$, respectively. Data in each case has been scaled to account for the different propagation path lengths so that contour increments are the same in each figure. The distribution of phase error for propagation at $\alpha=45^{\circ}$ (fig. 2a) is highly localized and shows the largest phase errors, while the cases at $\alpha=135^{\circ}, 153.4^{\circ}$ (figs, $2 c, d$ ) show distributions somewhat elongated in the flow direction and much less phase errors (see also figure 3 ).

The (spatial) r.m.s. phase error normalized by the value for the vertical propagation $\left(\alpha=90^{\circ}\right)$ is plotted in figure 3 . In accord with the above qualitative indications, the r.m.s. phase error attains a maximum for the propagation along the vortical structures $\left(\alpha_{\max } \simeq 45^{\circ}\right.$ or $\left.-135^{\circ}\right)$ and a minimum at an angle approx- 


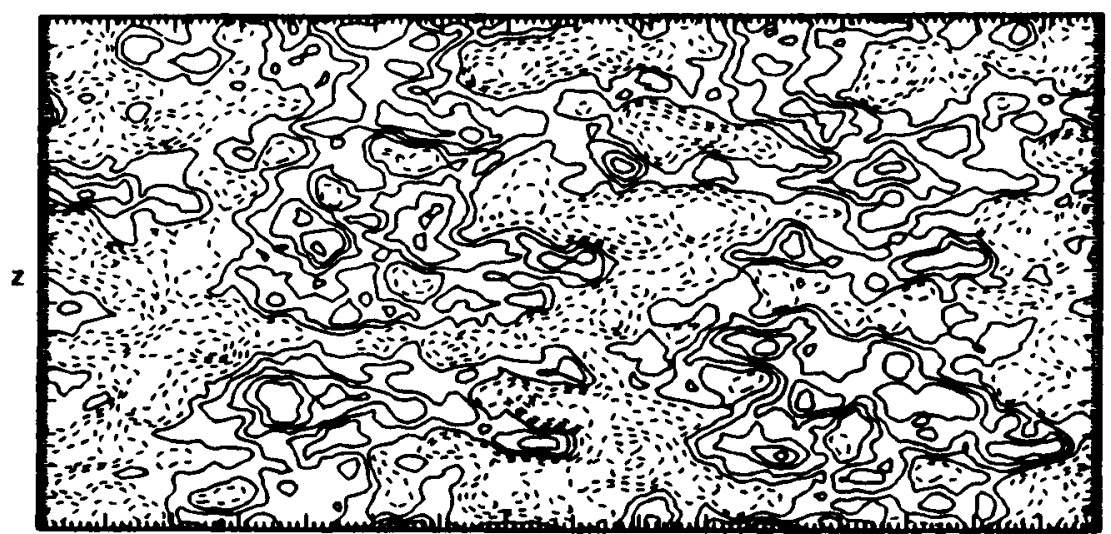

(a)

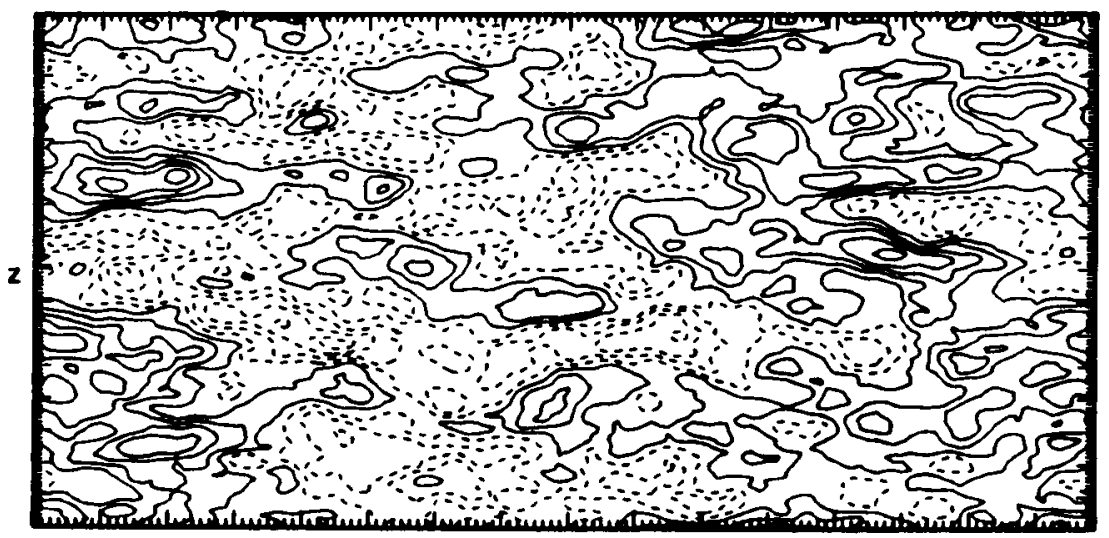

(b)

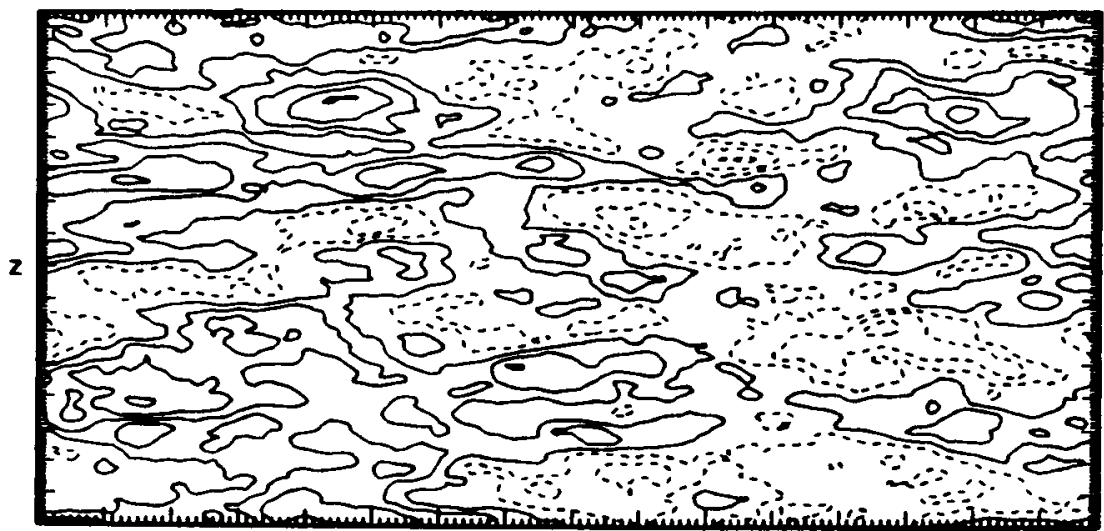

(c)

Figure 2 $(a-c)$. For caption see next page. 


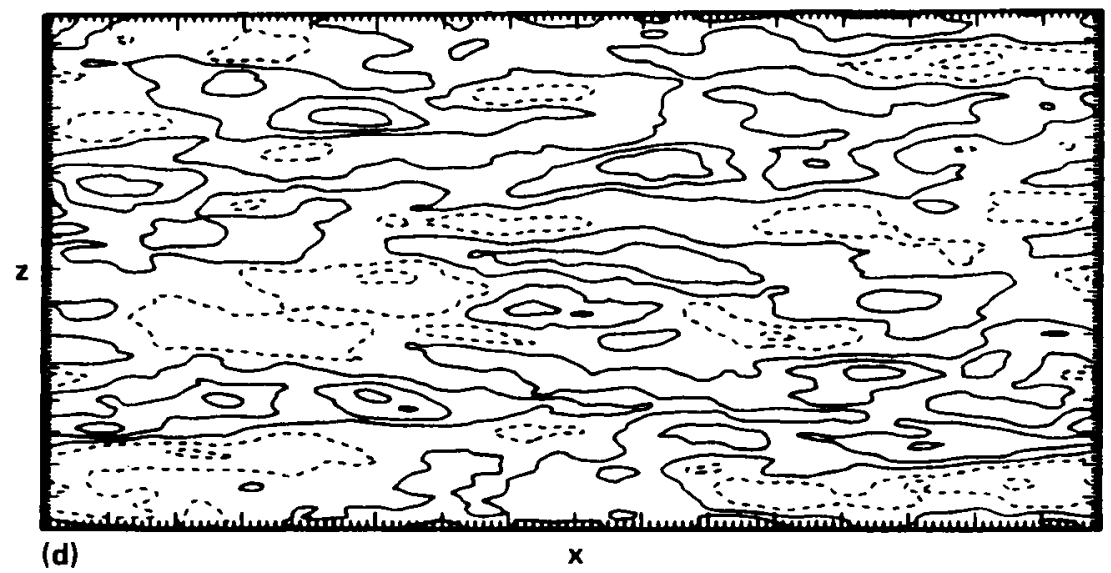

Figure 2. Contours of the phase error $\Delta \phi$ on the horizontal $x z$-plane for propagation through homogeneous shear flow at various angles $\alpha$ : (a) $45^{\circ} ;(b) 90^{\circ} ;(c)$ $135^{\circ} ;(d) 153.4^{\circ}$.

imately normal to them $\left(\alpha_{\min } \simeq 150^{\circ}\right.$ or $\left.-30^{\circ}\right)$. The maximum r.m.s. phase error at $\alpha_{\max }$ is about twice the minimum value at $\alpha_{\min }$. Consequently, these results confirm that the scalar fluctuations are concentrated in regions aligned with the vortical structures and are elongated in that direction.

\subsection{Physical model for scalar fluctuations}

The above results indicate that the distribution of scalar fluctuations is directly influenced by the vortical structures, namely the numerous hairpin vortices (Rogers \& Moin 1987). A simple model relating scalar fluctuations to the vortical structures is shown in figure 4. The flow induced by an 'upright' hairpin has a region of $n^{\prime}<0$ between and above its legs, and regions of $n^{\prime}>0$ outside and below its legs. This is a direct consequence of the dominant role of the hairpin vortices in inducing the scalar fluctuations: 'cold' fluid $\left(n^{\prime}<0\right)$ is brought up between the hairpin legs, while 'hot' fluid $\left(n^{\prime}>0\right)$ is pulled down outside the legs. Conversely, an 'inverted' hairpin has a region of $n^{\prime}>0$ between and below its legs and regions of $n^{\prime}<0$ outside and above its legs. The importance of this scalar transport by vortical structures to the scalar flux was discussed by Rogers et al. (1986).

\subsection{Two-point correlations}

The feasibility of the above model for scalar fluctuations induced by vortical structures can be tested by examining the two-point cross-correlations of scalar and vorticity fluctuations. It is suggested from the conceptual model for generation of scalar fluctuation shown in figure 4 that there should be a strong correlation between vorticity and scalar fluctuation at a separation in the spanwise direction $r_{z}$ of about half the average spacing $\Lambda_{z}$ of the hairpin legs.

In figures 5 and 6 , the spanwise cross-correlations of vorticity and scalar made 


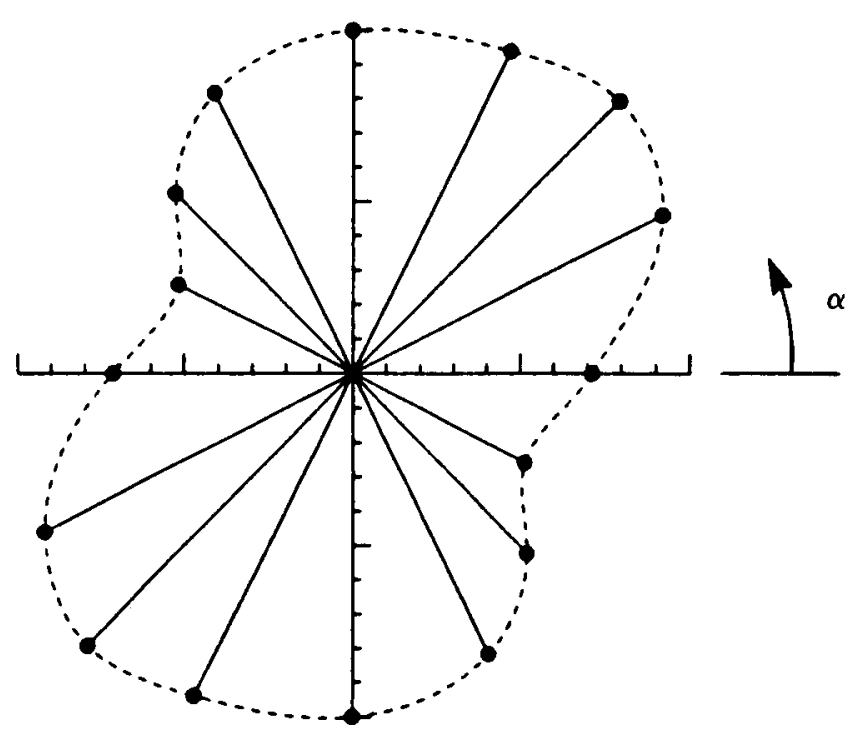

FigurE 3. Dependence of r.m.s. phase error on the angle of beam propagation. The r.m.s. values are normalized by the value for the vertical propagation $\left(\alpha=90^{\circ}\right)$.

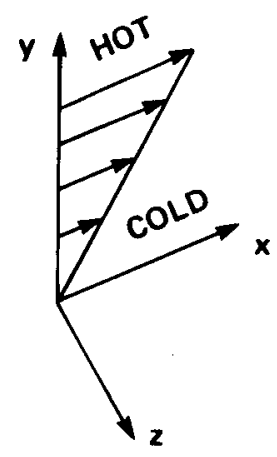

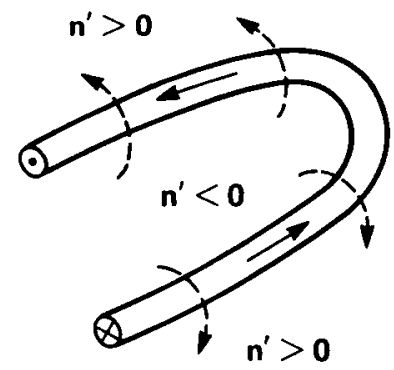

UPRIGHT HAIRPIN

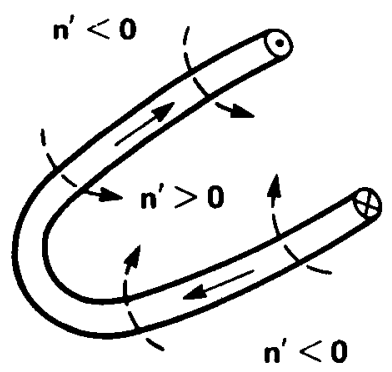

INVERTED HAIRPIN

FIGURE 4. Schematic of a conceptual picture showing the scalar fluctuations induced by the 'upright' and 'inverted' hairpin vortices. The arrows with solid line indicate vorticity and the arrows with broken line describe how the induced flow field transports passive scalar. 


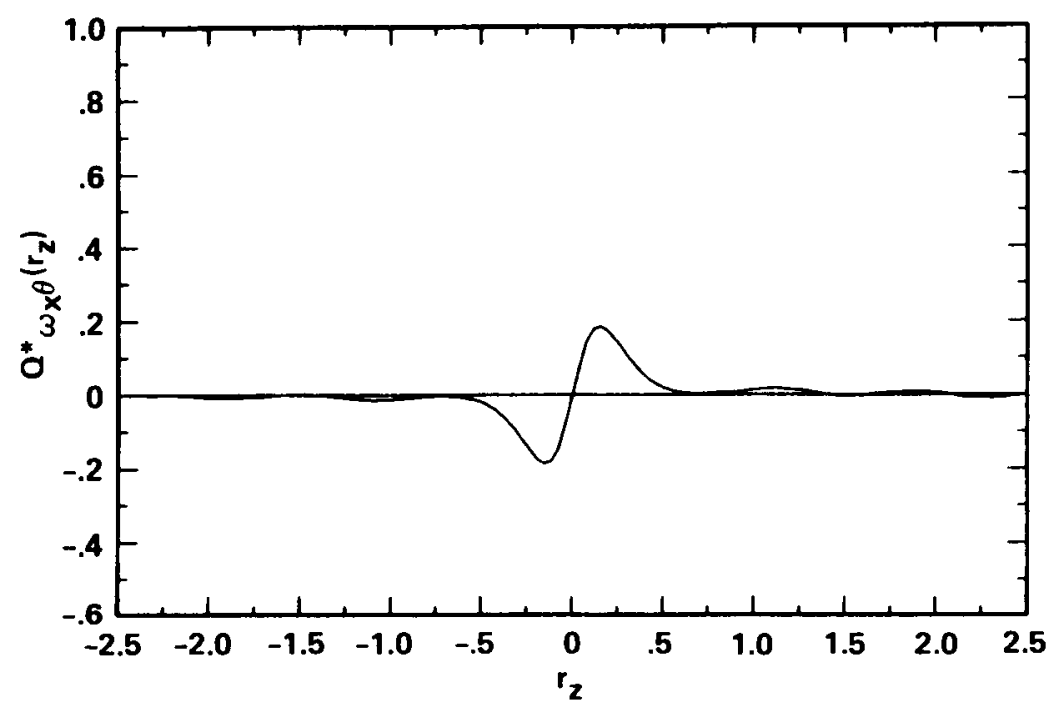

Figure 5. Two-point spanwise correlation $Q_{\omega_{z} \theta}^{*}\left(r_{z}\right)$ of $\omega_{x}$ and $\theta$, showing a pair of peak and valley at distances half the hairpin-leg spacing: $\left|r_{z}\right| \simeq \frac{1}{2} \Lambda_{z}$.

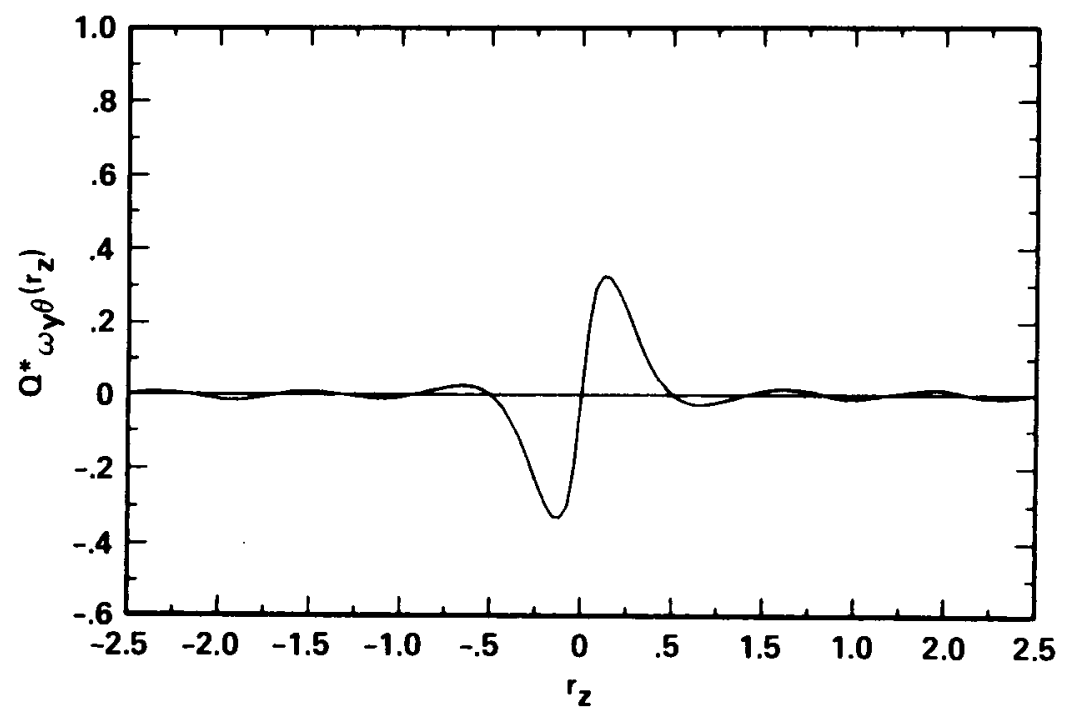

FIGURE 6. Two-point spanwise correlation $Q_{\omega_{y} \theta}^{*}\left(r_{z}\right)$ of $\omega_{y}$ and $\theta$, showing a pair of peak and valley at distances half the hairpin-leg spacing: $\left|r_{z}\right| \simeq \frac{1}{2} \Lambda_{z}$. 
dimensionless by the respective r.m.s. values $\omega_{i}^{\prime}$ and $\theta^{\prime}$ are shown:

$$
\left.Q_{\omega_{i} \theta}^{*}(\mathbf{r})=\frac{\overline{\omega_{i}(\mathbf{x}) \theta(\mathbf{x}+\mathbf{r})}}{\omega_{i}^{\prime} \theta^{\prime}} \quad \text { (no sum on } i\right)
$$

where the overbar denotes the statistical average taken over the three-dimensional space. It is evident that there exists a fair amount of correlation between the scalar and vorticity fluctuations. Because the hairpins are oriented (on the average) at $45^{\circ}$ in the $x y$-plane, both $\omega_{x}$ and $\omega_{y}$ correlate well with $\theta$. For positive spanwise separations $\left(r_{z}>0\right)$ the correlation is positive $Q_{\omega_{i} \theta}^{*}\left(r_{z}\right)>0$, whereas for negative span wise separations $\left(r_{z}<0\right)$ the correlation is negative $Q_{\omega_{i} \theta}^{*}\left(r_{z}\right)<0$. Notice that each leg of the upright as well as inverted hairpins contributes to the correlations in the same manner.

The two-point spanwise correlations $Q_{\omega_{z} \theta}^{*}\left(r_{z}\right)$ and $Q_{\omega_{y} \theta}^{*}\left(r_{z}\right)$ in the figures indeed show a pair of distinctive peak and valley antisymmetrically. The distance between the peak and valley corresponds to the average spacing between the hairpin legs $\Lambda_{z} \simeq 0.23$, estimated from the two-point auto-correlation of vorticity. This is entirely consistent with the physical picture of scalar-fluctuation generation sketched in figure 4. The correlations computed for a field at an earlier time, $S t=8$ (not shown), are nearly identical.

\section{Conclusions}

The phase distortion induced in a coherent optical beam by turbulent fluctuations in a homogeneus shear flow is highly anisotropic, i.e. sensitive to the direction of propagation. This is a result of intense scalar (i.e. index-of-refraction) fluctuations in elongated regions which are inclined to the mean flow similar to the vortical structures (hairpin vortices). A conceptual model is proposed for scalar fluctuations produced by the hairpin eddies, which are characteristic of homogeneous shear flow. This concept is supported by the two-point vorticity-scalar correlations which show a pair of maximum and minimum at spanwise distances which correspond to onehalf the spacing between the hairpin legs.

The importance of large-scale vortical structures and the associated scalar distribution to optical distortion has been established. It would be of fundamental interest to investigate the relative importance of small- and large-scale turbulent structures to phase distortion in propagation through turbulent shear flow. A study of probability density functions of the phase error and two-point correlations of vorticity and scalar gradient would also be of interest. An examination of the effects of inhomogenity could be carried out by using databases from direct numerical simulation of turbulent boundary layers and mixing layers.

Helpful discussions were held with L. Hesselink, M. M. Rogers and S. K. Lele. Support by AFOSR Project 2307Y1, through the Air Force Weapons Laboratory, Albuquerque, New Mexico, is gratefully acknowledged. 


\section{REFERENCES}

Baxter, M. R., Truman, C. R. \& Masson, B. S. 1988 Predicting the optical quality of supersonic shear layers. AIAA Paper 88-2771.

BORN, M. \& WOLF, E. 1975 Principles of Optics: Electromagnetic Theory of Propagation, Interference and Diffraction of Light. 5th edn. Pergamon: Oxford, England.

Clark, T. T., Truman, C. R. \& Masson, B. S. 1988 Prediction of optical phase degradation through a turbulent shear flow. AIAA Paper 88-3664.

Goodman, J. W. 1985 Statistical Optics. Wiley-Interscience: New York.

KELLER, J. B. 1954 Geometrical acoustics. I. The theory of weak shock waves. J. Appl. Phys. 25, 938-947.

LiepmanN, H. W. 1979 The rise and fall of ideas in turbulence. Amer. Sci. 67, 221-228.

Monin, A. S. \& Yaglom, A. M. 1975 Statistical Fluid Mechanics: Mechanics of Turbulence, Vol. 2, MIT Press: Cambridge, Mass.

Rogers, M. M. \& MoIN, P. 1987 The structure of the vorticity field in homogeneous turbulent flows. J. Fluid Mech. 176, 33-66.

Rogers, M. M., MoIn, P. \& REYNolds, W. C. 1986 The structure and modeling of the hydrodynamic and passive scalar fields in homogeneous turbulent shear flow. Dept. Mech. Engng. Rep. TF-25, Stanford University: Stanford, California.

Sutton, G. W. 1969 Effect of turbulent fluctuations in an optically active fluid medium. A.I.A.A. J. 7, 1737-1743.

TATARSKII, V. I. 1971 The effects of the turbulent atmosphere on wave propagation. NSF Rep. TT 68-50464, Nat. Tech. Info. Service (NTIS), U.S. Dept. of Commerce. [Translated from the Russian original (1967, «NAUKA》: Moscow) by Israel Program for Scientific Translations (IPST) Staff.] 